Perspektiven der Analytischen Philosophie Perspectives in Analytical Philosophy

\author{
Herausgegeben von \\ Georg Meggle und Julian Nida-Rümelin
}

Band 1

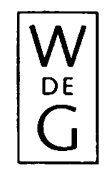

Walter de Gruyter · Berlin · New York

1994 


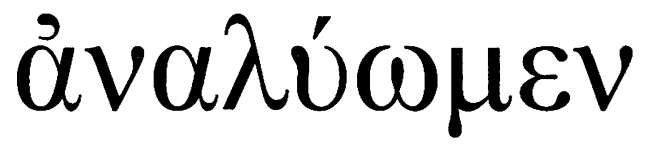 \\ Analyomen 1}

Proceedings of the 1st Conference
"Perspectives in Analytical Philosophy"

Edited by

Georg Meggle and Ulla Wessels

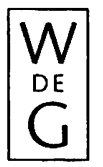

Walter de Gruyter - Berlin - New York 
(0) Printed on acid-free paper which falls within the guidelines of the ANSI to ensure permanence and durability.

\section{Libary of Congress Cataloging-in-Publication Data}

Analyōmen 1 = Analyomen $1:$ proceedings of the 1 st conference "perspectives in analytical philosophy" / edited by Georg Meggle and Ulla Wessels.

p. $\quad \mathrm{cm}$. - (Perspectives in analytical philosophy $=$ Perspektiven der analytischen Philosophie; Bd. 1)

English and German.

Proceedings of the first congress of the Gesellschaft für Analytische Philosophie, Oct. 9-12, 1991, Saarbrücken, Germany.

Includes bibliographical references and index.

ISBN 3-11-013581-7 (alk. paper)

1. Analysis (Philosophy)-Congresses. I. Meggle, Georg. II. Wessels, Ulla. III. Gesellschaft für Analytische Philosophie. IV. Series: Perspectives in analytical philosophy ; Bd. 1. B808.5.A49 1993

$146^{\prime} .4-\mathrm{dc} 20$

\section{Die Deutsche Bibliotbek - CIP-Einheitsaufnabme}

Analyōmen : proceedings of the 1st conference "perspectives in analytical philosophy" = Analyomen / ed. by Georg Meggle and Ulla Wessels. - Berlin ; New York : de Gruyter.

NE: Meggle, Georg [Hrsg.]

1 (1994)

(Perspectives in analytical philosophy ; Bd. 1)

ISBN 3-11-013581-7

NE: Perspektiven der analytischen Philosophie

(C) Copyright 1994 by Walter de Gruyter \& Co., D-10785 Berlin

All rights reserved, including those of translation into foreign languages. No part of this book may be reproduced or transmitted in any form or by any means, electronic or mechanical, including photocopy, recording, or any information storage and retrieval system, without permission in writing form the publisher.

Printed in Germany

Typesetting and Printing: Arthur Collignon $\mathrm{GmbH}$, Berlin

Binding: Lüderitz \& Bauer, Berlin 


\section{Contents}

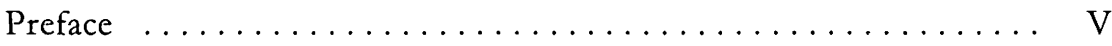

List of Contributors $\ldots \ldots \ldots \ldots \ldots \ldots \ldots \ldots \ldots \ldots \ldots \ldots$

Introduction

GeOrg Henrik von Wright

Analytische Philosophie - eine historisch-kritische Betrachtung ... 3

\section{Logic}

Jacqueline Brunning

A Proposal for a Reconstruction of Peirce's 1870 Notation of Relatives 33

Christoph Fehige

The Limit Assumption in Deontic (and Prohairetic) Logic ..... 42

Bertram Kienzle

Ereignisse einer syrakusischen Nacht $\ldots \ldots \ldots \ldots \ldots \ldots \ldots . \ldots 7$

Reinhard Kleinknecht

Theory of Descriptions and Truth-Set Semantics ........... 68

Helmut Linneweber-Lammerskitten

A Survey of the Derivability of Important Implicative Principles in Alternative Systems of Propositional Logic ............. 76

INGOLF MAX

Generalized Variable Functors Representing Paraconsistent Operators 88 Uwe Meixner

Nichttarskische Semantik der modalen Aussagenlogik . . . . . . 98

Ulrich Metschl

Necessity and Provability .......................... 103

UlRich NorTMANN

Does Aristotele's Modal Logic Rest on Metaphysical Assumptions? 115

Gerhard Schurz

Eine logische Analyse des Sein-Sollen-Problems ........... 126 
Peter Steinacker

Die epistemische Komponente nichtklassischer Funktoren.

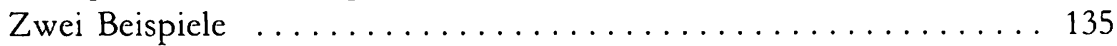

WERner Stelzner

Relevanz, Konsistenz \& Entailment ................. 146

Horst Wessel

Alternative Logiken und empirische Wissenschaften .......... 168

\section{Epistemology}

\section{Stefan Gosepath}

On the Rationality of Beliefs ....................... 179

Michael Kober

Wittgenstein and Forms of Scepticism ................ 187

Dirk Koppelberg

Naturalistische Erkenntnistheorien und Probleme der Normativität 198

Franz von Kutschera

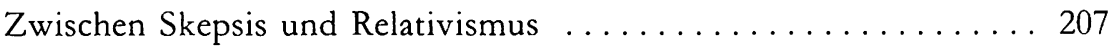

Ulrich Majer

Ein konstruktiver Begriff der Wahrheit .............. 225

Thomas Mormann

Cassirer's Problem and Geometrical Aspects of Epistemology .... 241

SANDRa B. Rosenthal

Charles Peirce and the Issue of Foundations . . . . . . . . . 251

Käthe Trettin

Logische Formalisierungen und Evidenz . . . . . . . . . . . . . . . 259

Klaus Volkert

Anschauliche Unmöglichkeit versus logische Unmöglichkeit zur erkenntnistheoretischen Diskussion über die nicht-euklidische Geometrie .................................. 266

III. Philosophy of Science - Historical and Systematical

Thomas Bartelborth

Wissenschaftlicher Realismus und approximative Theorien.

Zur Explikation des wissenschaftlichen Realismus 
Andreas Bartels

Intertheoretische Begriffsrelationen und Referenzrahmen in der Physik ............................... 286

Adam Grobler

Justification of the Empirical Basis: The Popperian vs. the Inductivist

Conception of Rationality

Michael Heidelberger

Alternative Interpretationen der Repräsentationstheorie der Messung 310

Paul Hoyningen-Huene

Emergenz versus Reduktion .................... 324

EkATERINI KaleRI

Zur strukturellen Analogie zwischen hermeneutischem Interpretieren und wissenschaftlichem Theoretisieren ............... 333

Alfons Keupink

Statistical Ambiguity and Inductive Inconsistencies .......... 345

Hans Kraml

Roger Bacon's Theory of the Rainbow as a Turning Point in the Pre-Galilean Theory of Science ................... 353

Hans Scheibe

The Divorce Between the Sciences and the Humanities ....... 362

Gerhard Terton

Methodologische Erklärungsmodelle aus heuristischer Sicht .... 379

Max UrCHS

Causal Priority. Towards a Logic of Event Causation .......... 386

HANS WestMeyer

Der strukturalistische Ansatz in der Theoretischen Psychologie . . . 397

IV. Philosophy of Language

C. Anthony Anderson

Degrees of Intentionality $\ldots \ldots \ldots \ldots \ldots \ldots \ldots \ldots \ldots \ldots \ldots \ldots \ldots \ldots$

Elke Brendel

The Liar Paradox: An Extensional Alternative to the Situation

Semantics Approach .......................... 421

Filip Buekens

Keeping Track of Pierre's Mind. A Davidsonian Solution to Kripke's Puzzle About Belief .......................... 434 
Christoph JäGER

Hybride singuläre Sinne und präsentische Propositionen ....... 444

Matthias Kaufmann

Ockham und Davidson über die Wahrheit .............. 453

M. Thomas Liske

Mögliche Welten bei David Lewis und bei Kripke ........... 464

Ana Marostica

The Semiotic and the Semantic Conception of Truth ......... 474

Felix Mühlhölzer \& Marianne Emödy

Über eine mögliche Inkonsistenz in Chomskys Auffassung von Sprachregeln ............................ 481

Albert Newen

How to Fix the Reference of 'that' in Demonstrative Utterances ... 493

Ulrich Pardey

Identität und Reflexivität . . . . . . . . . . . . . . . . . . . . . . . . . . . . 509

Peter Philipp

PU $₫$ 293: Private und öffentliche Käfer . . . . . . . . . . 520

RICHARD RAATZSCH

„Die gemeinsame menschliche Handlungsweise“ (PU 206) . . . . . 529

Artur Rojszczak

Über die Korrespondenz von Tarskis Definition der Wahrheit . . . . 539

EDMUND RUNGgALdiER

Referenz und „zeitliche Teile“ .................. 544

EIKE von SAVIGNY

Stücke einer Definition des Wahrheitsbegriffs für bedeutungsvolle Äußerungen ........................... 550

Hans Julius Schneider

Wie systematisch kann eine Theorie der Bedeutung sein? . . . . . . 564

Oliver Scholz

Zum Status allgemeiner Verstehensprinizien $\ldots \ldots \ldots \ldots \ldots \ldots . \ldots 74$

KLaus Wuttich

Bedingungen für den Sprechakt des Lügens $\ldots \ldots \ldots \ldots \ldots \ldots 58$

V. Philosophy of Mind

Antoni Gomila

Punctate Minds and Fodor's Theory of Content ........... 605 
Michael ter Hark

Cognitive Science, Propositional Attitudes and the Debate Between

Russell and Wittgenstein .................... 612

Heinz-Dieter Heckmann

Can Personal Identity Be Analysed in Terms of Relations of

(Non-branching) Continuity? . . . . . . . . . . . . . . . . . 618

Geert Keil

Is the Computational Metaphor of Mind Intentionalistic or

Naturalistic? . . . . . . . . . . . . . . . . . . . . . . . . . . . . . . . 629

Andreas Kemmerling

Mentale Repräsentationen - gibt es sie? . . . . . . . . . . . . . 640

Peter Lanz

Funktionalismus und sensorisches Bewußtsein . . . . . . . . . . 648

Georg Meggle

Zukünftige Dienstage . . . . . . . . . . . . . . . . . 660

Thomas Metzinger

Subjectivity and Mental Representation ............... 668

Audun Øfsti

Searle, Leibniz and „The First Person“. A Note on the Epilogue of

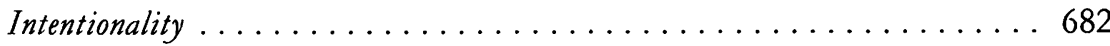

SVEN RosenkRANZ

A Review of Eccle's Arguments for Dualist-Interactionism . . . . . 689

Katia SAPORITI

Fodors naturalistischer Begriff der Bedeutung . . . . . . . . . . . 695

\section{Practical Philosophy}

Antonella Corradini

Abtreibung und das Prinzip der Doppelwirkung . . . . . . . . . . 707

Rafael Ferber

Moral Judgements as Descriptions of Institutional Facts . . . . . 719

Martina Herrmann

Wie beschafft man sich moralische Intuitionen? . . . . . . . . 730

Helmut F. Kaplan

Ethik, Leid und Mitleid . . . . . . . . . . . . . . . . . . 737

Matthias KettNer

„Geltungsansprüche“ 
HANS LENK

Zwischen Metaphysik und normativen Interpretationskonstrukten -

die Wiederkehr praktischer Fragen in der analytischen Philosophie 761

Christoph Lumer

Was ist eine triftige Moralbegründung? . . . . . . . . . . . . 785

JULIAN NIDA-RÜMELIN

Ethischer Kognitivismus ohne Intuitionen . . . . . . . . . . . . . . 797

Klaus Peter Rippe

Artenschutz als Problem der Praktischen Ethik . . . . . . . . . . . 805

BEATE RÖSSLER

Quotierung als moralisches Problem ................ 818

ECKARD ROLF

Emotionen und Handlungen . . . . . . . . . . . . . . 832

Peter Schaber

Externe Handlungsgründe . . . . . . . . . . . . . . . . . . . . 842

SABINE Thürmel

Ethische Aspekte der virtuellen Realität . . . . . . . . . . . . 850

Jean-Claude Wolf

Utilitarismus, Verantwortung und kriminelle Versuche ........ 856

VII. Miscellanea

Winfried Franzen

„Die wahre und einzige Methode der Philosophie ist also die analytische ...". Der junge Herder über die Philosophie und ihr Verhältnis zur Sprache

Dietfried Gerhardus

Die Rolle von Probe und Etikett in Goodmans Theorie der Exemplifikation

Christian Kanzian

Der Begriff „Koinzidenz“ in der Mereologie

Harald KöHL

Selbstbestimmung und Verzweiflung . . . . . . . . . . . . . 899

WINFRIED LÖFFLER

Modale Versionen des ontologischen Arguments für die Existenz Gottes 
GeBHARD LÖHR

Kann der Glaube an Gott die Frage nach dem Sinn des Lebens

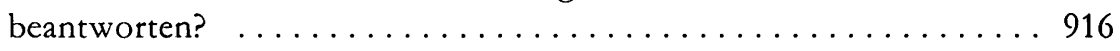

Kuno Lórenz

Was ist der Mensch? - Auch eine Frage der analytischen Philosophie 927 Jaroslav Peregrin

Formalisation of Language as a Means of Philosophical Analysis . . 939 Rolf W. Puster

Sprachanalytisches Argumentieren bei John Locke . . . . . . . . . . 946 Antonio Zilhao

Ludwig Wittgenstein and Edmund Husserl ............. 956

Appendices

Appendix One

Satzung der Gesellschaft für Analytische Pbilosopbie (GAP) . . . . . 967

Appendix Two

Georg Meggle: Bericht des Präsidenten vor der 1. Mitgliederversammlung der Gesellschaft für Analytische Philosophie (GAP) am 10.10 .1991 in Saarbrücken . . . . . . . . . . . . . . . . 972

Ergänzungen und Nachträge vom 10. 8. 1992 . . . . . . . . . . 977

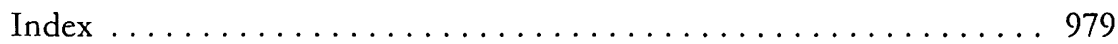




\title{
Ethischer Kognitivismus ohne Intuitionen
}

\author{
JULIAN NIDA-RÜMELIN
}

Ethischer Kognitivismus (EK) ist eine Theorie zweiter Ordnung. Diese Theorie besagt, daß moralische Äußerungen wahrheitsfähig sind. Sie besagt nicht, daß jede moralische Äußerung wahrheitsfähig sei. Moralische Äußerungen, die einen Wahrheitsanspruch haben, nennen wir i. F. moralische Urteile. Eine ethische Theorie erster Ordnung ist dadurch gekennzeichnet, daß sie moralische Urteile impliziert. Moralische Urteile, die einen Begründungsanspruch unter Rekurs auf eine ethische Theorie erster Ordnung erheben, nennen wir ethische Urteile. Es gibt zwei Typen moralischer und ethischer Urteile: Verpflichtungs- und Werturteile, bzw. normative und axiologische Urteile. Dementsprechend gibt es zwei Arten von EK: EK bezogen auf normative und EK bezogen auf axiologische Urteile, oder kurz: normativer EK und axiologischer EK. Normativer EK beinhaltet die Existenzbehauptung: Es gibt mindestens ein ethisches oder moralisches Verpflichtungsurteil, das wahrheitsfähig ist.

Die Theorie des ethischen Non-Kognitivismus behauptet das Gegenteil: es gibt kein moralisches/ethisches Urteil das wahrheitsfähig ist. Der ethische Non-Kognitivismus hat bis in die Gegenwart hinein, die analytische praktische Philosophie dominiert, auch wenn es - beginnend mit Victor Kraft - eine Reihe prominenter Dissidenten gegeben hat, zu der u. a. Stephen Toulmin, Kurt Baier, Bernard Gert, Elisabeth Anscombe, Hilary Putnam, Gerald Dworkin und Tom Nagel zählen.

Die Stärke des ethischen Non-Kognitivismus beruht auf der Konjunktion zweier Argumente:

(1) dem Argument des naturalistischen Fehlschlusses, bzw. dem openquestion-Argument (Moore 1903, chap. 1)

und

(2) dem Argument der ontologischen Absonderlichkeit (Mackie 1977, chap. 1)

Während das erste Argument eine naturalistische Version des EK ausschließt, besteht das zweite in einer reductio ad absurdum für die 
intuitionistische Variante. Sofern Naturalismus und Intuitionismus als eine vollständige Disjunktion des EK gelten, steht es schlecht um den EK und die Bereitschaft auch eher abwegige non-kognitivistische Theorien zweiter Ordnung zu akzeptieren, wie sie besonders für eine frühe Phase der analytischen Philosophie typisch war, wird verständlich.

Ich möchte i. F. für einen EK ohne moralische Intuitionen, oder anders formuliert - für einen EK argumentieren, der im engeren Sinne nicht intuitionistisch ist. $\mathrm{Da}$ ich das Argument des naturalistischen Fehlschlusses, trotz der Schwächen seiner ursprünglichen Darstellung bei George Edward Moore, im Kern für zutreffend halte, stehen dann nur nicht-naturalistische und zugleich nicht-intuitionistische Varianten des EK offen.

Der ethische Intuitionismus ist die fundamentalistische nicht-naturalistische Variante des EK. Der Fundamentalismus (im Sinne von foundationalism) ist eine Theorie zweiter Ordnung, die besagt, daß Theorien erster Ordnung über einen bestimmten Gegenstandsbereich erstens aus fundamentalen, nicht begründungsfähigen, aber selbstevidenten (oder unmittelbaren) Propositionen und zweitens aus Propositionen bestehen, die sich aus den fundamentalen, ableiten' lassen. Man kann den generellen Fundamentalismus eines Descartes, Leibniz oder des frühen Carnap von einem partiellen unterscheiden, der sich auf spezifische Gegenstandsbereiche erstreckt (etwa bei Aristoteles).

Entsprechend der angenommenen Natur der Beziehung von fundamentalen und zu begründenden Propositionen ergeben sich drei Varianten des Fundamentalismus: (1) Platonismus (Theorie der Formen, Urteilen aufgrund der unmittelbaren Erfassung (Schau) der Formen), (2) Induktivismus (logische Wahrscheinlichkeit einer Hypothese aufgrund unmittelbar gegebener Daten) und (3) Deduktivismus (Logische Ableitung der Sätze einer Theorie aus unmittelbar einsichtigen Annahmen, bzw. Axiomen).

Nach der Art der fundamentalen Propositionen ergeben sich ebenfalls unterschiedliche Typen des Fundamentalismus, es lassen sich (1) phänomenalistische (die fundamentalen Propositionen beziehen sich auf Sinnesdaten), (2) realistische (die fundamentalen Propositionen beziehen sich auf externe Gegenstände und ihre Eigenschaften) und (3) idealistische (die fundamentalen Propositionen beziehen sich auf ideale Entitäten, Formen, Begriffe, Ideen) Versionen des Fundamentalismus unterscheiden.

Nicht jede Form des ethischen Fundamentalismus ist zugleich intuitionistisch. Der kantische Apriorismus, für den das moralische Gesetz einen apriorischen und begründenden Status hat, ist nicht intuitionistisch. Das moralische Gesetz wird nicht intuitiv erfaßt, sondern ist ein Postulat der 
Rationalität. Die Theorie erhebt, wie alle rationalen Ethiken, (cf. NidaRümelin 1992) den Anspruch einer außermoralischen Rechtfertigung unbeschadet dessen, daß die, gemeine sittliche Vernunfterkenntnis' mit den philosophischen Prinzipien praktischer Rationalität übereinstimmt. Soweit man die ethische Theorie Kants isoliert betrachtet, ist das moralische Gesetz eine fundamentale Proposition im oben eingeführten Sinne: es dient der Begründung nicht-fundamentaler Propositionen, zum Teil unter Zuhilfenahme weiterer axiomatischer Setzungen ohne selbst eine Begründung im Rahmen der ethischen Theorie zu erfahren. Der Unterschied zu intuitionistischen Theorien besteht darin, daß sich der Gültigkeitsanspruch des moralischen Gesetzes nicht auf die (unmittelbare) moralische Intuition stützt.

Intuitionistische Theorien haben dagegen bei aller inhaltlichen Vielfalt eines gemeinsam: Bestimmte Propositionen der Theorie, seien es Werturteile, wie bei George Edward Moore, aber auch bei Max Scheler und Nicolai Hartmann, generelle Verpflichtungsurteile wie bei David Ross oder singuläre Verpflichtungsurteile, wie im französischen Existenzialismus, aber auch bei Herbert Prichard, sind fundamental und stützen ihren Geltungsanspruch auf die (unmittelbare) moralische Intuition.

A.: Varianten des ethischen Intuitionismus
I. normativ
a) singuläre Handlung (action token)
b) generische Handlung (action type)
c) ethisches Prinzip
11. axiologisch
a) singuläre Instantiierung
b) generische Entitäten
c) universelle Werte

Für teleologische Varianten des cthischen Intuitionismus gilt das Primat des Guten vor dem Richtigen: Verpflichtungsurteile sind durch Werturteile begründet und bestimmte Typen von Werturteilen sind fundamental. Deontologische Varianten des ethischen Intuitionismus im weiteren Sinne bilden das Supplement, d. h. für sie gilt nicht das Primat des Guten vor dem Rechten. Deontologische Varianten des ethischen Intuitionismus im engeren Sinne behaupten das Primat des Rechten vor dem Guten. Für deontologische Varianten des eth. Intuitionismus im weiteren Sinne haben neben Werturteile auch bestimmte Typen von Verpflichtungsurteilen fundamentalen Status. Für deontologische Varianten des ethischen Intuitionismus im engeren Sinne haben ausschließlich bestimmte Typen von Verpflichtungsurteilen fundamentalen Status. Teleologische und starkdeontologische Varianten lassen sich daher jeweils als normativer oder 
axiologischer Intuitionismus charakterisieren, während schwach-deontologische Varianten eine Kombination von normativem und axiologischem Intuitionismus darstellen.

Einige Zuordnungen können diese Typologie verdeutlichen:

B.: Zuordnungen zur Typologie A

Ia: Sartre, Prichard

Ib: Ross

Ic: Sidgwick

IIa,b: Moore

IIc: Bentham

Allen intuitionistischen Ethiken gemeinsam ist die Auszeichnung bestimmter moralischer/ethischer Propositionen als fundamental. Intuitionistischen ethischen Theorien ist daher eine besondere Art Abgeschlossenheit zu eigen. Im Gegensatz etwa zu (ebenfalls fundamentalistischen) rationalen Ethiken, die Kriterien praktischer Rationalität oder apriorische Konsistenzbedingungen, zugrunde legen, bleiben die fundamentalen, begründenden Propositionen intuitionistischer Ethiken innerhalb des moralischen Bereichs. Moralische Erkenntnis ist im ethischen Intuitionismus in gewisser Weise autonom: Die Begründungskette ethischer Propositionen endet nicht außerhalb, sondern innerhalb der Ethik in einer Klasse nicht mehr begründungsfähiger fundamentaler ethischer Propositionen, die selbst nicht mehr begründungsbedürftig sind. Eine spezielle Form (für fundamentale Propositionen) nicht begründungsbedürftig zu sein, ist, selbstrechtfertigend zu sein, etwa im Sinne Sidgwicks, für den ethische Prinzipien dann selbstrechtfertigend sind, wenn sie vier Bedingungen erfüllen: durchsichtig und genau (clear and precise), bei Reflektion einleuchtend (evident upon reflection), wechselseitig konsistent (mutually consistent) und allgemein anerkannt (generally accepted) (Sidgwick 1907, 338 ff.).

Diese Gemeinsamkeit intuitionistischer Ethiken darf aber den Blick darauf nicht verstellen, daß moralische Intuitionen für die verschiedenen Typen intuitionistischer Ethik eine unterschiedliche Rolle spielen. Nur für intuitionistische Ethiken vom Typ Ia gilt, daß moralischen Intuitionen eine unmittelbare Geltung als Verpflichtungsgrund zukommt. Die anderen Versionen des normativen Intuitionismus revidieren (und korrigieren ggf.) moralische Intuitionen nach dem Maßstab der (intuitiv) als moralisch zulässig oder geboten ausgezeichneten generischen Handlungen (im Falle Ib) oder der (intuitiv akzeptierten) ethischen Kriterien (im Falle Ic). Dieser Unterschied kommt auch darin zum Ausdruck, daß die intuitionistische Ethik vom Typ Ia zur Theorielosigkeit tendiert (was besonders an den 
existenzialistischen Ethiken deutlich wird), während die Typen Ib und erst recht Ic ethische Theoriebildung nicht nur als möglich, sondern als notwendig zur angemessenen moralischen Urteilsbildung erachten.

Das Argument der ontologischen Absonderlichkeit läßt sich nur dann gegen den Intuitionismus verwenden, wenn eine zusätzliche metaphysische Prämisse hinzutritt: Die intuitive Erfassung der jeweils fundamentalen Propositionen der Theorie wird korrespondenztheoretisch interpretiert. Diese Interpretation ist allerdings nur für axiologische Varianten des Intuitionismus naheliegend. Das Werturteil, ,Freundschaft ist etwas intrinsisch Gutes', wird analog zu dem Tatsachenurteil ,Wiese ist etwas Grünes als Zuordnung einer realen Eigenschaft zu einem Gegenstand interpretiert. Die Ontologie enthält nun neben Entitäten, wie Farben auch Werteigenschaften. Diese Anreicherung allein erscheint manchen Kritikern schon als ausreichend, um den Intuitionismus zurückzuweisen (Mackie 1977). Ergänzt wird diese Kritik bisweilen mit dem Hinweis auf das Fehlen eines moralischen Sinnesorgans, das für diese spezielle Wahrnehmungsfähigkeit moralischer Eigenschaften zuständig sein könnte.

Das Argument der ontologischen Absonderlichkeit ist in der gleichen Weise auf den normativen Typ des ethischen Intuitionismus nicht anwendbar, denn es setzt voraus, ein Verpflichtungsurteil als Zuschreibung einer Eigenschaft zu interpretieren. Welcher Entität wird welche Eigenschaft etwa durch das Verpflichtungsurteil ,Hans sollte Gretl helfen` zugeordnet? Des generischen Prozesses des Gretlhelfens durch Hans, die Eigenschaft des Gesollt-seins? Aber auch für die axiologischen Varianten des ethischen Intuitionismus überzeugt das Argument der ontologischen Absonderlichkeit nicht, denn auch im außermoralischen Bereich ist die Ontologie einer korrespondenztheoretischen Interpretation von Propositionen notorisch unklar - dabei gibt es alte Probleme, wie die der sog. sekundären Qualitäten und neue, wie die der realistischen Interpretation der Modelle der theoretischen Physik.

Das entscheidende Argument gegen den ethischen Intuitionismus scheint mir nicht ontologischer, sondern epistemologischer Natur zu sein: genausowenig, wie es eine sichere theoriefreie Beobachtungsbasis der Naturwissenschaften gibt, so gibt auch keinen ethikfreien Fundus moralischer Intuitionen. Moralische Intuitionen bilden sich in einer komplexen Wechselwirkung von generellen meist nicht expliziten Kriterien und Einzelfallbetrachtungen in einem theoretischen Kontext, der empirisches und normatives Hintergrundwissen umfaßt, heraus und sind weder geeignet ethische Propositionen von hohem Allgemeinheitsgrad als Axiome einer ethischen Theorie zu begründen, noch experimenta crucis bereitzustellen, 
die als einzige Instanz über das Scheitern oder die Bewährung einer Theorie entscheiden. Die wissenschafts- und erkenntnistheoretischen Entsprcchungen der verschiedenen Formen des ethischen Intuitionismus, der kritische Rationalismus des frühen Popper oder (je nach Begründungsrelation) der positivistische Induktivismus des frühen Carnap für die a-Versionen, der frühneuzeitliche Rationalismus für die c-Versionen und Hobbes'sche operative Zwischenlösungen für die b-Versionen haben sich allesamt als unhaltbar herausgestellt. Eine Epistemologie, die Kohärenz und Systematisierung in den Mittelpunkt stellt, ist als Metatheorie auch der Ethik angemessener. Sie ist mit einer Vielfalt von ontologischen Positionen, darunter auch der metaphysisch-realistischen, vereinbar, aber sie löst sich vom Fundamentalismus und damit vom Privileg moralischer Intuitionen.

Schon die Bandbreite intuitionistischer Theorien bezüglich der Frage, welche Art moralischer Intuitionen die fundamentalen ethischen Propositionen jeweils bilden, kann man als Hinweis auf ein kohärenztheoretisches Verständnis ethischer Begründung auffassen. Eine kohärenztheoretische Epistemologie entkoppelt Begründung und Inferenz, sei diese induktiv oder deduktiv. Eine ethische Proposition ist begründet, heißt, sie paßt sich gut in einen systematischen Zusammenhang ein, der den Corpus zentraler moralischer Haltungen und Urteile (axiologische und normative), seien sie (eher) genereller oder (eher) singulärer Art, erfaßt und notwendige Revisionen so vornimmt, daß sie in das Überzeugungssystem im Kontext des moralischen und nicht-moralischen Hintergrundswissens gut integrierbar sind. Begründung ist damit keine ,lineare' Relation, die neben dem Abbruch in vermeintlichen Fundamentalpropositionen nur den unendlichen Regreß oder den Zirkel zuläßt. Logisch-deduktive Ableitung und ev. induktive (statistische) Bestätigungsrelationen spielen für die Systematisierung eine wichtige Rolle, aber sie sind nicht isomorph zur Begründungsrelation. Wie genau eine holistische und kohärentistische Epistemologie aussieht und ob sich eine solche in voller Allgemeinheit entwickeln läßt, kann und muß hier (nicht nur aus Zeitgründen) offenbleiben (cf. Rescher 1979, 1980). Es genügt zu sehen, daß eine Alternative zum Fundamentalismus auch in der Ethik denkbar ist. Damit ist zwar eine Ethik ohne Intuitionen i. e. S. denkbar, aber es bleibt die Frage: Kann eine solche Ethik kognitivistisch sein? M. a.W. läßt sich Kognitivismus, Non-Naturalismus, Anti-Intuitionismus und Kohärentismus zu einer ethischen Theorie zweiter Ordnung verbinden?

Die traditionellen Formen des ethischen Kognitivismus - Rationalismus, Intuitionismus und Naturalismus - schließen eine solche Verbindung aus. Der erste Kandidat ist der ethische Konstruktivismus, eine Theorie 
zweiter Ordnung, die in der letzten Dekade besonders durch den Einfluß John Rawls' Bedeutung erlangt hat (Rawls 1971, 1980). In der hier gebrauchten Terminologie können wir ethischen Konstruktivismus durch zwei Thesen definieren: Erstens, es gibt moralische (nicht-natürliche) Tatsachen (= zutreffende moralische Sachverhalte) und zweitens, moralische Tatsachen werden durch Kohärenz konstituiert. Der ethische Konstruktivismus verbindet also epistemischen Kohärentismus und Kohärenztheorie moralischer Wahrheit. Er kann in einer universalistischen und einer kontextualistischen, bzw. relativistischen Variante auftreten - Rawls selbst scheint zwischen beiden Formen unentschieden, was seine Vereinnahmung durch Richard Rorty auf der einen und Ronald Dworkin auf der anderen Seite möglich macht. Mit dem Konstruktivismus liegt nun eine kognitivistische Ethik ohne Intuitionen vor, er verbindet Kognitivismus, NonNaturalismus, Anti-Intuitionismus und Kohärentismus zu einer ethischen Theorie zweiter Ordnung. Wenn man die Parallele zur allgemeinen Erkenntnis- und Wissenschaftstheorie zieht, bildet der kontextualistische Konstruktivismus das ethische Gegenstück zu den Immanenztheorien Thomas Kuhns und Paul Feyerabends, während dem universalistischen Konstruktivismus eine Wahrheitstheorie zugrundeliegt, die im ,logischen Idealismus' Michael Dummetts eine Parallele hat.

Der Konstruktivismus ist mit folgender Objektivitätsthese allerdings nicht vereinbar: Es ist immer möglich, daß eine wohlbegründete ethische Proposition falsch ist. Evidenz und Wahrheit darf auch unter idealen Bedingungen nicht identifiziert werden. Wenn die Objektivitätsthese zutrifft, dann ist eine epistemische Wahrheitsdefinition grundsätzlich inadäquat und damit ließe sich auch der ethische Konstruktivismus als kognitivistische Ethik zweiter Ordnung nicht aufrechterhalten.

Wenn man diese Objektivitätsthese akzeptiert, bleiben zwei Varianten: schwacher und metaphysischer Objektivismus. Beide sind mit epistemischem Kohärentismus vereinbar und kommen daher ohne fundamentale moralische Intuitionen aus. Während der schwache Objektivismus über die Objektivitätsthese nicht hinausgeht, verknüpft der metaphysische Objektivismus diese These mit einer Korrespondenztheorie moralischer Wahrheit, was eine Welt theorien- und sprachunabhängiger Tatsachen voraussetzt.

Fazit:

(1) Die nach wie vor verbreitete These, daß Kognitivismus und NonNaturalismus zusammen ethischen Intuitionismus implizieren, ist nicht haltbar. 
(2) Die Ersetzung des epistemischen Fundamentalismus in der Ethik durch einen epistemischen Kohärentismus erlaubt vier Typen nicht-naturalistischer und nicht-intuitionistischer, aber doch kognitivistischer Ethiken:

(i) Kontextualistischer Konstruktivismus

(ii) Universalistischer Konstruktivismus

(iii) Schwacher Objektivismus

(iv) Metaphysischer Objektivismus

\section{Literatur}

Anscombe, Gertrude Elisabeth M., 1958, Modern Moral Philosophy. Philosopby 23, $1-19$.

Baier, Kurt, 1958, The Moral Point of View, Ithaca.

Bentham, Jeremy, 1843, Deontology, London-Edinburgh.

Dummett, Michael, 1958/9, Truth. Proceedings of the Aristotelian Society.

Dworkin, Gerald, 1978, Taking Rights Seriously, Harvard.

Gert, Bernard, 1966, The Moral Rules. A New Foundation of Morality, New York.

Gert, Bernard, 1988, Morality. A New Justification, Oxford.

Hartmann, Nicolai, 1926, Ethik, Berlin 1926.

Kraft, Victor, 1937, Die Grundlagen einer wissenschaftlichen Wertlebre, Wien.

Mackie, John Leslie, 1977, Ethics. Inventing Right and Wrong, Harmondsworth.

Moore, George Edward, 1903, Principia Ethica, Cambridge.

Nagel, Thomas, 1986, The View from Nowbere, Oxford.

Nida-Rümelin, 1992, Rationale Ethik. In Geschichte der neueren Ethik, Bd. 2, hrsg. Annemarie Pieper, Tübingen, Kap. 22.

Prichard, Herbert, 1949, Moral Obligation, Oxford.

Putnam, Hilary, 1978, Meaning and the Moral Sciences, London.

Rescher, Nicolas, 1979, Cognitive Systematization. A Systems-Theoretic Approach to a Coherentist Theory of Knowledge, Oxford.

Rescher, Nicolas, 1980, Induction. An Essay on the Justification of Inductive Reasoning, Oxford.

Rorty, Richard, 1991, The Priority of Democracy to Philosophy. In Objectivity, Relativism, and Truth. Philosophical Papers, Bd. 1, Cambridge.

Ross, David, 1930, The Right and the Good, Oxford.

Scheler, Max, 1913, Der Formalismus in der Etbik und die materiale Wertetbik, Halle.

Sidgwick, Henry, 1907, Methods of Ethics, Chicago.

Toulmin, Stephen, 1950, Reason in Ethics, Cambridge. 